\title{
A Branch-Type TE/TM Wave Splitter Using a Light-Guiding Metal Line
}

\author{
Tomohide Yamazaki, Student Member, IEEE, Junji Yamauchi, Member, IEEE, Member, OSA, \\ and Hisamatsu Nakano, Fellow, IEEE
}

\begin{abstract}
The three-dimensional (3-D) beam-propagation method is applied to the analysis of a novel transverse electric/ transverse magnetic (TE/TM) wave splitter using a light-guiding metal (Ag) line with an embedded dielectric $\left(\mathrm{SiO}_{2}\right)$ waveguide. Before analyzing the splitter, a mode converter with a tapered metal is designed to smoothly convert the guided mode of an embedded waveguide into the surface plasmon-polariton (SPP) mode, and vice versa. After demonstrating the effectiveness of a converter consisting of a two-step linear taper, the performance of the splitter is evaluated. To obtain a high coupling efficiency, the wavefront mismatch of the SPP mode is compensated for. In addition, a TE-pass polarizer is added to the TE output waveguide, reducing the undesirable $T M$ wave. In contrast to the long device length of a conventional branch-type splitter $(>1000 \mu \mathrm{m})$, the present splitter is found to have a noticeably short device length of less than $200 \mu \mathrm{m}$, although a loss of about $3 \mathrm{~dB}$ is observed for the TM wave. The crosstalk and the extinction ratio are, respectively, evaluated to be less than $-15 \mathrm{~dB}$ and more than $15 \mathrm{~dB}$ for a branching angle of $16 \pm 2^{\circ}$ at wavelengths of 1.31 and $1.55 \mu \mathrm{m}$.
\end{abstract}

Index Terms-Beam-propagation method (BPM), light-guiding metal line, mode converter, surface plasmon polaritons (SPP), TE/TM wave splitter.

\section{INTRODUCTION}

A WAVEGUIDE transverse electric/transverse magnetic (TE/TM) wave splitter is one of the essential components in integrated optics, such as polarization diversity receivers for coherent transmission systems [1] and polarization-independent wavelength filters [2]. Some investigations have been focused on the splitter consisting of a glass waveguide [3]-[11], which has the advantage of easiness of fabrication [12]. These splitters are roughly divided into two types, i.e., directional coupler type [3]-[7] and branch type [8]-[11].

A directional-coupler-type splitter utilizes the difference between the coupling lengths of the TE and TM waves. Note that the coupling length is sensitive to variation in the separation length between the two waveguides placed in parallel. Therefore, this type of splitter generally has a narrow bandwidth property. On the other hand, a branch-type splitter essentially operates over a wide range of wavelengths. The drawback of this type of splitter is a long device length of usually more than $1000 \mu \mathrm{m}$ [8]-[11]. A reduction in the device length requires

Manuscript received May 17, 2006; revised September 8, 2006. This work was supported in part by the Takahashi Industrial and Economic Research Foundation.

The authors are with the Faculty of Engineering, Hosei University, Koganei, Tokyo 184-8584, Japan (e-mail: tomohide.yamazaki.wh@gs-eng.hosei.ac.jp). Digital Object Identifier 10.1109/JLT.2006.889656 a larger branching angle, resulting in a lower extinction ratio. Consequently, it is difficult to realize a wide bandwidth property with a short device length.

To overcome this drawback, we have recently proposed a novel branch-type splitter using a light-guiding metal line [13], which is also called a "surface plasmon waveguide" [14]-[16]. Our idea comes from the fact that the strong field confinement of the surface plasmon-polariton (SPP) mode [17]-[19] permits an increase in a branching angle while maintaining a reasonable extinction ratio. This means that the splitter has the possibility of operating over a wide range of wavelengths with a short device length, although the TM wave inherently incurs some loss due to the field absorption in the metal.

The purpose of this paper is to reveal the properties of the splitter using the light-guiding metal line by the threedimensional (3-D) beam-propagation method (BPM). We particularly pay attention to the improvement in the TE/TM wave splitting properties.

To smoothly convert the guided mode of an embedded waveguide into the SPP mode and vice versa, we first determine the shape of a mode converter using a tapered metal. It is demonstrated that the use of a converter consisting of a twostep linear taper leads to a high coupling efficiency of more than $90 \%$ with a short taper length of $75 \pm 15 \mu \mathrm{m}$ at a wavelength of $1.55 \mu \mathrm{m}$.

We next assess the performance of the splitter. After compensating for the wavefront mismatch of the SPP mode, we add a TE-pass polarizer to the TE output waveguide to reduce an undesirable TM wave. As a result, the crosstalk and the extinction ratio are, respectively, evaluated to be less than $-15 \mathrm{~dB}$ and more than $15 \mathrm{~dB}$ for a branching angle of $16 \pm 2^{\circ}$ at wavelengths of 1.31 and $1.55 \mu \mathrm{m}$, with a loss of about $3 \mathrm{~dB}$ being observed for the TM wave. In contrast to the long device length of the conventional branch-type splitter $(>1000 \mu \mathrm{m})$ [8]-[11], the present splitter is found to have a noticeably short device length of less than $200 \mu \mathrm{m}$.

\section{PREliminary InVESTIGATION}

Fig. 1 shows the basic configuration of the TE/TM wave splitter (an improved configuration will be discussed later). For the TE wave, the presence of the metal is almost negligible, so that the field mainly propagates through Waveguide \#1. For the TM wave, an uptapered metal near the input serves to smoothly convert the guided mode of an embedded waveguide into the bound SPP mode localized around the metaldielectric interface [17] (the so-called $a_{b}$ mode in [20]). The SPP mode is guided 


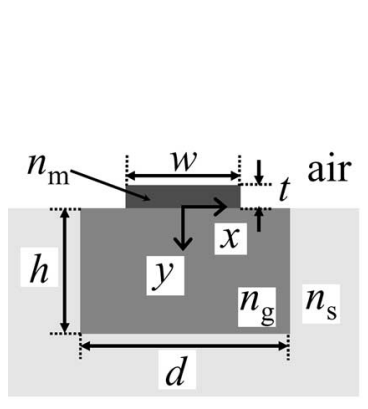

(a)

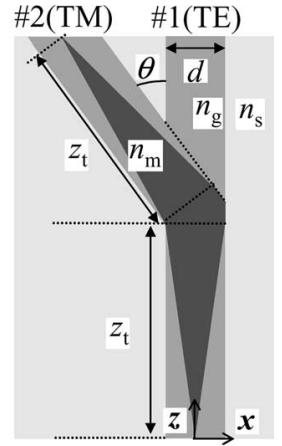

(b)
Fig. 1. Basic configuration of a TE/TM wave splitter $(h=2 \mu \mathrm{m}, d=3 \mu \mathrm{m})$. (a) Cross section. (b) Top view.

to Waveguide \#2 along the metal. In Waveguide \#2, the SPP mode is restored into the mode of the embedded waveguide by a downtapered metal. As a result, the TE and TM waves can be, respectively, extracted in Waveguides \#1 and \#2.

The refractive indexes of the dielectric core and substrate are set to be $n_{\mathrm{g}}=1.5$ and $n_{\mathrm{s}}=1.459$, respectively. The metal width is designated as $w$. The embedded waveguide without the metal $(w=0)$ supports only a single mode at both wavelengths of $\lambda=1.31 \mu \mathrm{m}$ and $\lambda=1.55 \mu \mathrm{m}$.

To analyze the splitter, we employ the semivectorial BPM based on the alternating-direction implicit scheme using the electric field [21], [22]. The sampling widths are fixed to be $\Delta x=0.05 \mu \mathrm{m}$ and $\Delta y=\Delta z=0.02 \mu \mathrm{m}$. The perfectly matched layer is placed at the edge of the computational region [19], [23].

It is known that the BPM often causes numerical instability [24]. Our calculation shows that the results become unstable as the field penetration into the metal increases with a subsequent large loss. This is observed, for example, when the imaginary part of the metal refractive index is intentionally taken to be small values, such as $n_{m}=0.14-j 4.0$. Fortunately, the instability is not observed when the refractive index of the metal is taken to be that of $\mathrm{Ag}$ or $\mathrm{Au}$ in the following analysis.

Before analyzing the splitter shown in Fig. 1, we compare the ohmic losses of the straight metal ( $\mathrm{Ag}$ and $\mathrm{Au}$ ) lines through the eigenmode analysis using the imaginary-distance (ID) BPM. For simplicity, we consider the 2-D structure $(w=$ $d=\infty)$. The operating wavelength is typically taken to be $\lambda=1.55 \mu \mathrm{m}$. The refractive indexes of $\mathrm{Ag}$ and $\mathrm{Au}$ are taken to be $n_{m}=0.14-j 11.2$ [25], [26] and $n_{m}=0.18-j 10.2$ [27], respectively. For $\mathrm{Ag}$, the calculation shows that the loss of the SPP mode asymptotically decreases as the metal thickness $t$ is increased. This is because the field becomes less confined to the metal with an increase in the thickness [19]. A further increase in the thickness leads to the fact that the loss for $t>0.1 \mu \mathrm{m}$ is almost constant. Similar behavior is also observed for $\mathrm{Au}$. Further calculation, however, shows that the loss of Ag with a specific thickness is smaller than that of Au. For example, the losses of $\mathrm{Ag}$ and $\mathrm{Au}$ for $t=0.2 \mu \mathrm{m}$ are calculated to be 13 and $22 \mathrm{~dB} / \mathrm{mm}$, respectively. We, therefore, adopt $\mathrm{Ag}$ of $t=0.2 \mu \mathrm{m}$ in the following analysis.

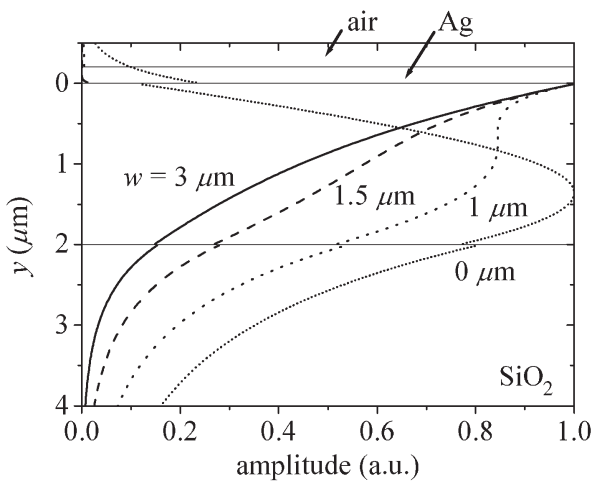

Fig. 2. Cross section of field distributions of the TM wave $(x=\Delta x / 2)$.

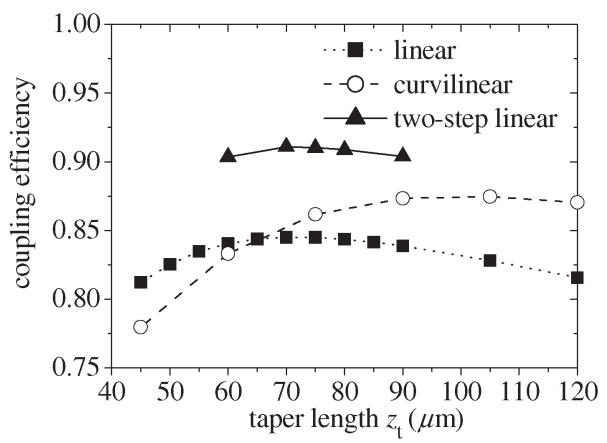

Fig. 3. Coupling efficiency against taper length.

\section{Mode Converter Using TAPered Metal}

We now design a mode converter using the metal (Ag) line in order to smoothly convert the guided mode of the embedded waveguide into the SPP mode. In the design, the knowledge of the eigenmode is of fundamental importance. Here, we evaluate the eigenmodes of the metal line shown in Fig. 1(a) for several metal widths using the ID-BPM [19], [28]-[30].

Fig. 2 shows the cross section of the field $\left(\left|E_{y}\right|\right)$ distributions observed at $x=\Delta x / 2$. The mode gradually changes from the guided mode of the embedded waveguide $(w=0)$ to the SPP mode when increasing the metal width (this fact also holds true for $\mathrm{Au} \mathrm{[17]-[19]).} \mathrm{Consequently,} \mathrm{the} \mathrm{tapered} \mathrm{metal} \mathrm{is}$ expected to smoothly convert the guided mode of the embedded waveguide into the SPP mode and vice versa.

We next assess the performance of the converter using the tapered metal by the propagating beam analysis. The metal is linearly or curvilinearly tapered in the propagating direction $z$. The change in the shape of the metal is expressed as follows:

$$
w(z)=w\left(z_{\mathrm{t}}\right)\left(\frac{z}{z_{\mathrm{t}}}\right)^{m}
$$

where $z_{\mathrm{t}}$ presents the taper length. The metal width at $z=z_{\mathrm{t}}$ is fixed to be $w\left(z_{\mathrm{t}}\right)=3 \mu \mathrm{m}$. The metal of $m=1$ is linearly tapered and that of $m>1$ is curvilinearly tapered.

Fig. 3 plots the coupling efficiencies of the linearly and curvilinearly $(m=2)$ tapered metals. We calculate the efficiency between the propagating field at $z=z_{\mathrm{t}}$ and the eigenmode field for $w=3 \mu \mathrm{m}$, in which the ohmic and radiation losses 


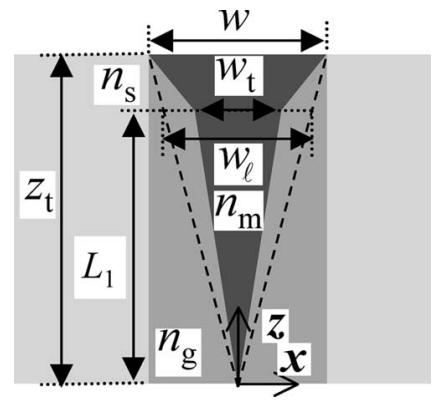

Fig. 4. Configuration of a mode converter consisting of a two-step linear taper.

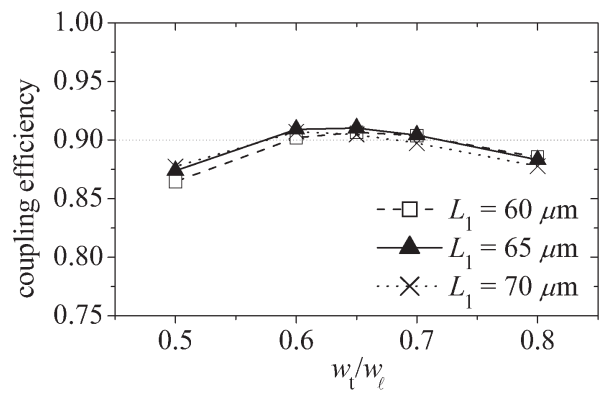

Fig. 5. Coupling efficiency against $w_{\mathrm{t}} / w_{\ell}$ at $z_{\mathrm{t}}=75 \mu \mathrm{m}$.

are taken into account. It should be noted that the ohmic loss increases with an increase in the metallic area [17], [19]. The curvilinearly tapered metal offers the low ohmic loss due to the small metallic area. However, the use of the short taper length gives rise to the rapid mode conversion with a subsequent increase in the radiation loss. As a result, a long taper length of $100 \mu \mathrm{m}$ is required to obtain the maximum efficiency $(\approx 87 \%)$. In contrast, the linearly tapered metal permits a short taper length of $70 \mu \mathrm{m}$ for obtaining the maximum efficiency $(\approx 85 \%)$. Unfortunately, the linearly tapered metal has the large metallic area, resulting in an increase in the ohmic loss. To reduce the losses, maintaining the short taper length, we introduce a two-step linear taper in the following.

Fig. 4 illustrates the mode converter consisting of the twostep linear taper with the metallic region being reduced. To obtain a high efficiency, we appropriately adjust the length of the first taper section $L_{1}$ and the width $w_{\mathrm{t}}$ at $z=L_{1}$. For reference, the shape of the linearly tapered metal is also illustrated by dashed lines in Fig. 4. The width at $z=L_{1}$ is denoted as $w_{l}$.

To investigate the effect of adjusting $L_{1}$ and $w_{\mathrm{t}}$, we analyze the converter for a specific taper length. As an example, the coupling efficiency against $w_{\mathrm{t}} / w_{l}$ at $z_{\mathrm{t}}=75 \mu \mathrm{m}$ is shown in Fig. 5. The efficiency is improved to be about $90 \%$ in a range of $0.6 \leq w_{\mathrm{t}} / w_{l} \leq 0.7$, when $L_{1}$ is chosen to be $65 \pm 5 \mu \mathrm{m}$.

The coupling efficiency is again calculated as a function of taper length, and the results are included in Fig. 3. A high efficiency of more than $90 \%$ is obtained over a wide range of $z_{\mathrm{t}}=75 \pm 15 \mu \mathrm{m}$. The advantage is that the length of the present tapered metal is substantially shorter than that of the curvilinearly tapered one. As a result, the configuration parameters are fixed to be $z_{\mathrm{t}}=75 \mu \mathrm{m}, L_{1}=65 \mu \mathrm{m}$, and $w_{\mathrm{t}} / w_{l}=0.65$.

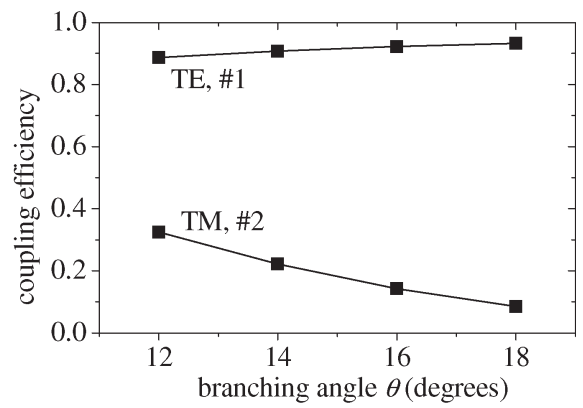

Fig. 6. Coupling efficiency against branching angle.

\section{TE/TM WAVE SPLitTER}

\section{A. Compensation for the Wavefront Mismatch of the SPP Mode}

Since the characteristics of the mode converter have been revealed, we next assess the performance of a splitter, which comprises the two mode converters with the two-step linear taper. Fig. 6 shows the coupling efficiencies of the TE and TM waves as a function of branching angle $\theta$. We calculate the efficiency between the propagating field at the output and the eigenmode field of the embedded waveguide. The efficiency of the TE wave is calculated to be about $90 \%$ for $12^{\circ} \leq \theta \leq 18^{\circ}$. In contrast, the efficiency of the TM wave is evaluated to be less than $32 \%$. One reason of the low efficiency is that the field is absorbed by the metal. The other is that the wavefront of the SPP mode cannot be sufficiently inclined to Waveguide \#2 and that the field tends to radiate toward the $z$-axis.

We now consider a technique for compensating for the wavefront mismatch of the SPP mode. Here, we notice that some compensation techniques have been studied in a bent waveguide without a metal [31]-[33]. For instance, the refractive index of the outside of the bend is reduced, while that of the inside is increased. This leads to inclination of the wavefront, since the local phase velocity in the low index region is faster than that in the high index region. Note that the effective index of the SPP mode increases with an increase in the metal width, resulting in a larger effective index than that of the guided mode in the waveguide without the metal [19]. Hence, we modify the shape of the metal to compensate for the wavefront mismatch of the SPP mode.

Fig. 7 illustrates a modified splitter, in which the metal of the outside of the bend is reduced, while that of the inside is increased. The wavefront of the SPP mode is adjusted by means of variations of the lengths of $a$ and $c$.

Fig. 8 shows the coupling efficiency for $\theta=14^{\circ}$ against length $c$. For reference, Fig. 8 also shows the length of $a$ for the maximum efficiency in each length of $c$. The efficiency is improved to be more than $54 \%$ in a range from $c=23 \mu \mathrm{m}$ to $c=30 \mu \mathrm{m}$.

We again evaluate the coupling efficiency against branching angle, as shown in Fig. 9. The lengths of $a$ and $c$ for each angle are chosen as exhibited in Table I. The efficiency is higher than that for $a=c=0$, as shown in Fig. 6, over a wide range of $12^{\circ} \leq \theta \leq 18^{\circ}$. It is also seen that the splitter maintains an efficiency of about $50 \%$ for $\theta=14 \pm 2^{\circ}$. For reference, Fig. 9 


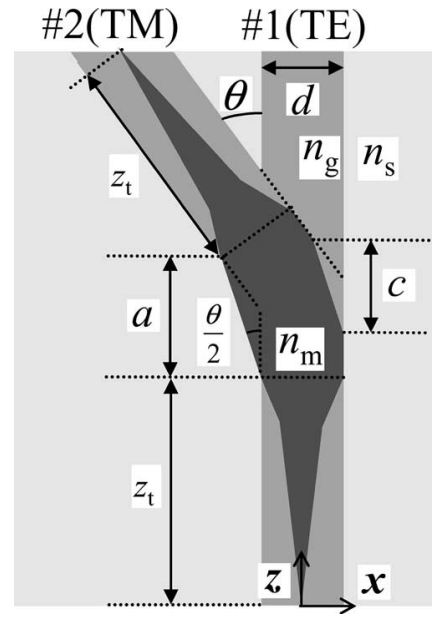

Fig. 7. Configuration of a modified splitter with wavefront compensation.

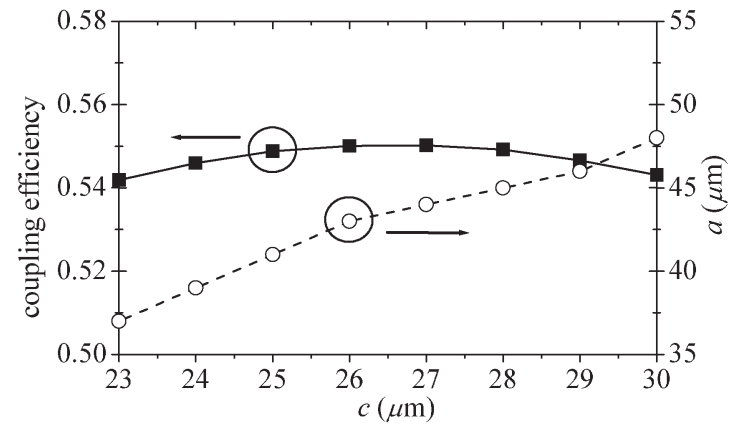

Fig. 8. Coupling efficiency and length $a$ against length $c\left(\theta=14^{\circ}\right)$.

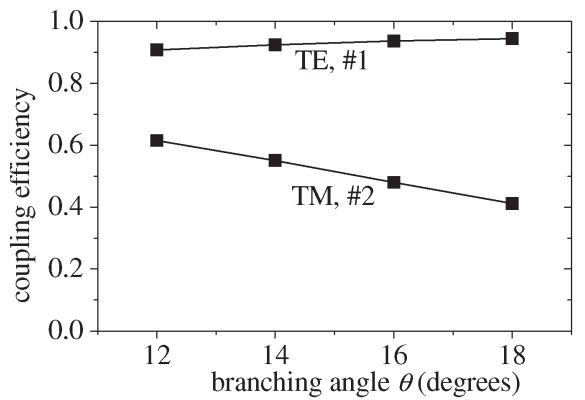

Fig. 9. Coupling efficiency against branching angle.

also shows the result of the TE wave. The efficiency is almost the same as that for $a=c=0$.

Figs. 10 and 11 show the crosstalk and the extinction ratio, which are defined by

$$
\begin{aligned}
& \mathrm{CT}_{\mathrm{TE}(\mathrm{TM})}=10 \log _{10}\left(C_{\mathrm{TE} \# 2(\mathrm{TM} \# 1)} / C_{\mathrm{TE} \# 1(\mathrm{TM} \# 2)}\right) \\
& \text { for } \mathrm{TE}(\mathrm{TM}) \text { wave } \\
& \mathrm{ER}_{\# 1(\# 2)}=10 \log _{10}\left(C_{\mathrm{TE} \# 1(\mathrm{TM} \# 2)} / C_{\mathrm{TM} \# 1(\mathrm{TE} \# 2)}\right) \\
& \text { in Waveguide \#1(\#2) }
\end{aligned}
$$

where $C$ is the coupling efficiency. The crosstalk of the TM wave is higher than that of the TE wave, while the extinction ratio in Waveguide \#1 is lower than that in Waveguide \#2. This is because part of the TM-wave field remains in Waveguide \#1.
TABLE I

LENGTHS $a$ AND $c$ FOR EACH BRANCHING ANGLE

\begin{tabular}{ccc}
\hline$\theta$ (degrees) & $a(\mu \mathrm{m})$ & $c(\mu \mathrm{m})$ \\
\hline 12 & 43 & 28 \\
\hline 14 & 44 & 27 \\
\hline 16 & 44 & 25 \\
\hline 18 & 42 & 22 \\
\hline
\end{tabular}

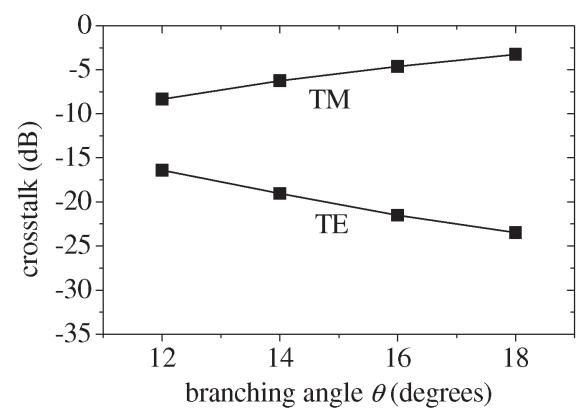

Fig. 10. Crosstalk against branching angle.

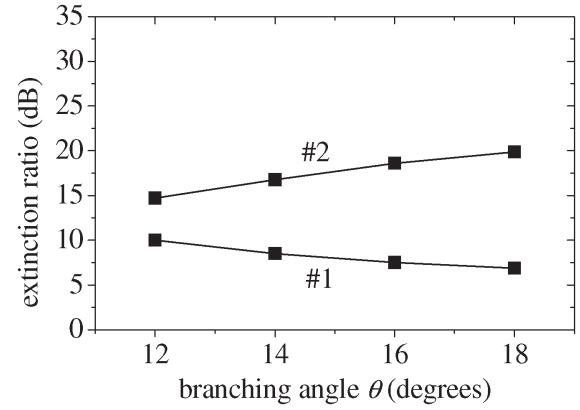

Fig. 11. Extinction ratio against branching angle.

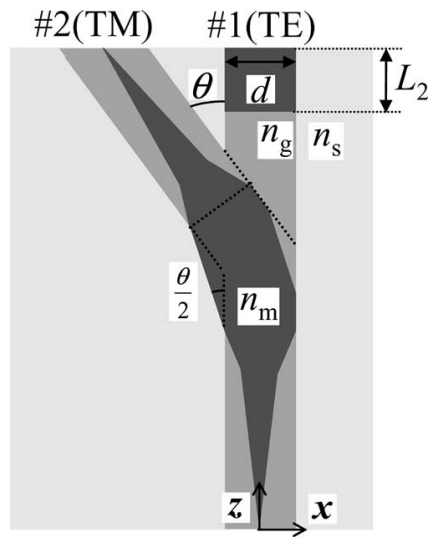

Fig. 12. Configuration of an improved splitter with a polarizer.

\section{B. Reduction in the TM-Wave Field in Waveguide \#1}

To reduce the remaining TM-wave field, we add a TE-pass polarizer to the output of Waveguide \#1. We should recall that the metal offers the ohmic loss, particularly for the TM wave. Hence, we load an additional metal on Waveguide \#1.

Fig. 12 illustrates the configuration of an improved splitter with the TE-pass polarizer. The polarizer length is designated as $L_{2}$. Since a branch-type splitter is expected to operate over a wide range of wavelengths, we consider the properties at not 


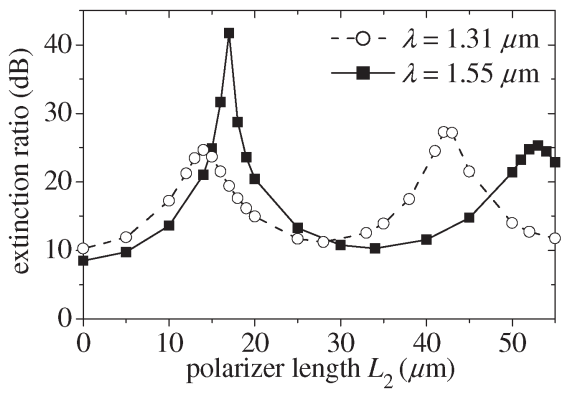

Fig. 13. Extinction ratio against polarizer length $\left(\theta=14^{\circ}\right)$.

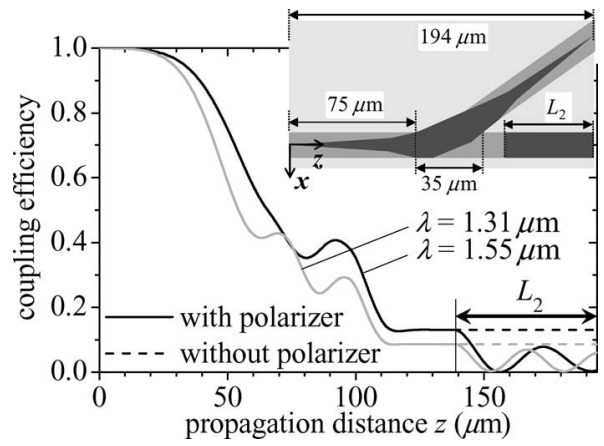

Fig. 14. Coupling efficiency of the TM wave along Waveguide \#1 $\left(L_{2}=\right.$ $55 \mu \mathrm{m})$.

only $\lambda=1.55 \mu \mathrm{m}$ but also at $\lambda=1.31 \mu \mathrm{m}$. The refractive index of the metal at $\lambda=1.31 \mu \mathrm{m}$ is calculated from the Drude model dielectric function $\left(n_{m}=0.1-j 9.46\right)$ [25].

Fig. 13 shows the extinction ratios at $\lambda=1.31 \mu \mathrm{m}$ and $\lambda=1.55 \mu \mathrm{m}$ for $\theta=14^{\circ}$ against polarizer length. The result for $L_{2}=0$ at $\lambda=1.55 \mu \mathrm{m}$ corresponds to that without the polarizer presented in Fig. 11. High extinction ratios of about $24 \mathrm{~dB}$ are obtained around $L_{2}=15 \mu \mathrm{m}$ at both wavelengths.

Fig. 13 also shows that the extinction ratio almost periodically oscillates against the polarizer length. To clarify this reason, we evaluate the coupling efficiency of the TM wave along Waveguide \#1, as shown in Fig. 14. For comparison, the results without the polarizer are also shown by broken lines (see the region designated as $L_{2}$ ). It is seen that the efficiency decreases as the field propagates, owing to the effect of the mode converter. In the region where the polarizer is placed, the efficiency tends to decrease with oscillation. The oscillation can be explained in terms of mode interference. At the junction between the embedded waveguide and the polarizer $(z \approx 140 \mu \mathrm{m})$, part of the guided-mode power of the embedded waveguide is converted into the guided-mode power of the polarizer (the overlap integral between the two guided modes is calculated to be about $50 \%$ at $\lambda=1.55 \mu \mathrm{m}$ ) and the remaining power into the power of radiation modes. This results in the coherent coupling between the guided mode and radiation modes with the subsequent oscillation. Consequently, the extinction ratio can be more than $20 \mathrm{~dB}$, provided that the polarizer length is chosen to be close to half the period of the oscillation, i.e., $L_{2}=15 \mu \mathrm{m}$.

We finally summarize the performance against the branching angle for $L_{2}=15 \mu \mathrm{m}$. Figs. 15-17 show the coupling efficien-

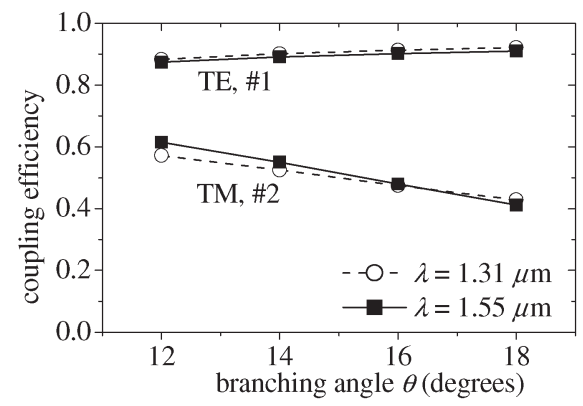

Fig. 15. Coupling efficiency against branching angle.

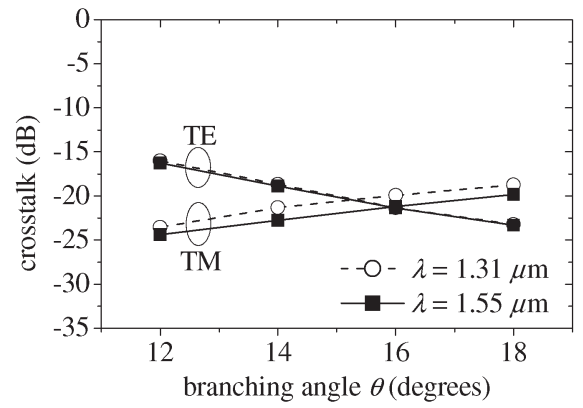

Fig. 16. Crosstalk against branching angle.

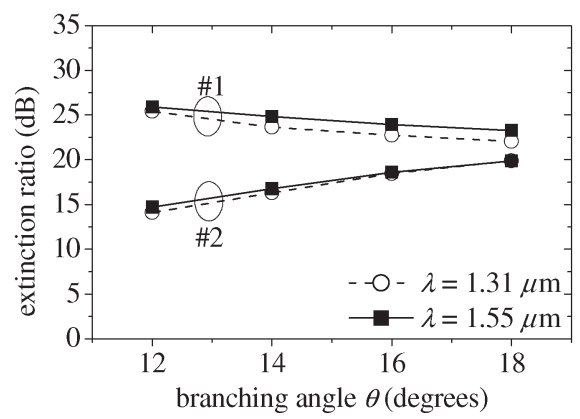

Fig. 17. Extinction ratio against branching angle.

cies, crosstalks, and extinction ratios at both $\lambda=1.31 \mu \mathrm{m}$ and $\lambda=1.55 \mu \mathrm{m}$, respectively. The efficiency of the TM wave and the extinction ratio in Waveguide \#2 at $\lambda=1.55 \mu \mathrm{m}$ are almost the same as those presented in Figs. 9 and 11. Similar splitting behavior at $\lambda=1.55 \mu \mathrm{m}$ is observed at $\lambda=1.31 \mu \mathrm{m}$. It is noteworthy that as compared with Figs. 10 and 11, the crosstalk of the TM wave and the extinction ratio in Waveguide \#1 are improved to be less than $-19 \mathrm{~dB}$ and more than $22 \mathrm{~dB}$ for $12^{\circ} \leq \theta \leq 18^{\circ}$, respectively. This leads to the fact that crosstalks of less than $-15 \mathrm{~dB}$ are obtained for the TE and TM waves and that extinction ratios of more than $15 \mathrm{~dB}$ are obtained in Waveguides \#1 and \#2 for $\theta=16 \pm 2^{\circ}$, with a loss of about $3 \mathrm{~dB}$ for the TM wave.

Typical field distributions of the TE and TM waves for $\theta=14^{\circ}$ at $\lambda=1.55 \mu \mathrm{m}$ are shown in Fig. 18(a) and (b), respectively. It is observed that the fields of the two waves are successfully separated into the Waveguides \#1 and \#2 around $z=200 \mu \mathrm{m}$. The device length is found to be remarkably shorter than that of the conventional branch-type splitter [8]-[11]. 

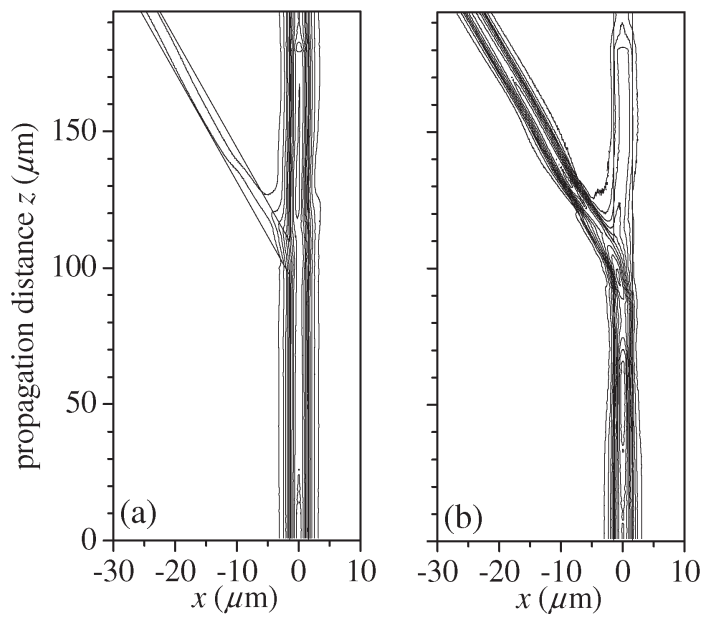

Fig. 18. Field distributions at $y=1 \mu \mathrm{m}$. (a) TE wave. (b) TM wave.

\section{Conclusion}

We have developed a novel branch-type TE/TM wave splitter using a light-guiding metal line. The 3-D BPM is applied to the analysis of the splitter. Before analyzing the splitter, a mode converter using a tapered metal has been designed in order to smoothly convert the guided mode of an embedded waveguide into the SPP mode, and vice versa. Numerical results show that a converter consisting of a two-step linear taper achieves a high coupling efficiency of more than $90 \%$ with a short taper length of $75 \pm 15 \mu \mathrm{m}$ at $\lambda=1.55 \mu \mathrm{m}$. We have next assessed the performance of the splitter. After compensating for the wavefront mismatch of the SPP mode with a subsequent increase in the efficiency in Waveguide \#2, a TE-pass polarizer is added to Waveguide \#1, reducing the undesirable TM wave. It is revealed that a crosstalk of less than $-15 \mathrm{~dB}$ and an extinction ratio of more than $15 \mathrm{~dB}$ are obtained for a branching angle of $16 \pm 2^{\circ}$ at $\lambda=1.31 \mu \mathrm{m}$ and $\lambda=1.55 \mu \mathrm{m}$, with a loss of about $3 \mathrm{~dB}$ being observed for the TM wave. The use of the lightguiding metal line contributes to a remarkably short device length of less than $200 \mu \mathrm{m}$.

\section{ACKNOWLEDGMENT}

The authors would like to thank H. Aono for his basic investigations of the present work.

\section{REFERENCES}

[1] V. Magnin, J. Harari, and D. Decoster, "Numerical study of polarizationselective side-illuminated pin photodetectors grown on InP substrate for hybridisation on silicon platform," Optoelectron., vol. 151, no. 3, pp. 171-176, 2004.

[2] W. Warzanskyj, F. Heismann, and R. C. Alferness, "Polarizationindependent electro-optically tunable narrow-band wavelength filter," Appl. Phys. Lett., vol. 53, no. 1, pp. 13-15, Jul. 1988.

[3] K. Thyagarajan, S. Diggavi, and A. K. Ghatak, "Design and analysis of a novel polarisation splitting directional coupler," Electron. Lett., vol. 24, no. 14 , pp. 869-870, Jul. 1988.

[4] —_, "Integrated-optic polarization-splitting directional coupler," Opt. Lett., vol. 14, no. 23, pp. 1333-1335, Dec. 1989.

[5] R. A. Betts and F. Lui, "Broadband polarisation splitting couplers in ionexchanged glass," Electron. Lett., vol. 26, no. 7, pp. 450-452, Mar. 1990.

[6] A. N. Miliou, R. Srivastava, and R. V. Ramaswamy, "A 1.3- $\mu$ m directional coupler polarization splitter by ion exchange," J. Lightw. Technol., vol. 11, no. 2, pp. 220-225, Feb. 1993.
[7] K. C. Lin, W. C. Chuang, and W. Y. Lee, "Proposal and analysis of an ultrashort directional-coupler polarization splitter with an NLC coupling layer,” J. Lightw. Technol., vol. 14, no. 11, pp. 2547-2553, Nov. 1996.

[8] Y. Shani, C. H. Henry, R. C. Kistler, R. F. Kazarinov, and K. J. Orlowsky, "Integrated optic adiabatic polarization splitter on silicon," Appl. Phys. Lett., vol. 56, no. 2, pp. 120-121, Jan. 1990.

[9] T. Mizumoto, N. Iwakiri, T. Kaneko, and Y. Naito, "Analytical and experimental study of waveguide optical polarization splitter with Langmuir-Blodgett cladding layer," J. Lightw. Technol., vol. 10, no. 12, pp. 1807-1813, Dec. 1992.

[10] R. M. de Ridder, A. F. M. Sander, A. Driessen, and J. H. J. Fluitman, "An integrated optic adiabatic TE/TM mode splitter on silicon," J. Lightw. Technol., vol. 11, no. 11, pp. 1806-1811, Nov. 1993.

[11] T. Hayakawa, S. Asakawa, and Y. Kokubun, "ARROW-B type polarization splitter with asymmetric Y-branch fabricated by a self-alignment process," J. Lightw. Technol., vol. 15, no. 7, pp. 1165-1170, Jul. 1997.

[12] M. Kawachi, "Silica waveguides on silicon and their application to integrated-optic components," Opt. Quantum Electron., vol. 22, no. 5, pp. 391-416, Sep. 1990.

[13] T. Yamazaki, H. Aono, J. Yamauchi, and H. Nakano, "BPM simulation of a branch-type TE/TM mode splitter using a light-guiding metal line," in Proc. OSA Integr. Photon. Res. Appl. Dig., 2005. IME3.

[14] T. Goto, Y. Katagiri, H. Fukuda, H. Shinojima, Y. Nakano, I. Kobayashi, and Y. Mitsuoka, "Propagation loss measurement for surface plasmonpolariton modes at metal waveguides on semiconductor substrates," Appl. Phys. Lett., vol. 84, no. 6, pp. 852-854, Feb. 2004.

[15] J. C. Weeber, Y. Lacroute, A. Dereux, E. Devaux, T. Ebbesen, C. Girard, M. U. González, and A. L. Baudrion, "Near-field characterization of Bragg mirrors engraved in surface plasmon waveguides," Phys. Rev. B, Condens. Matter, vol. 70, no. 23, p. 235 406, Dec. 2004.

[16] R. Zia, M. D. Selker, and M. L. Brongersma, "Leaky and bound modes of surface plasmon waveguides," Phys. Rev. B, Condens. Matter, vol. 71, no. 16, p. 165 431, Apr. 2005.

[17] T. Yamazaki, J. Yamauchi, J. Shibayama, and H. Nakano, "BPM simulation of a light-guiding metal line with an embedded dielectric core," in Proc. Numer. Simul. Optoelectron. Devices Conf., 2004, pp. 109-110. Paper ThD3.

[18] T. Yamazaki, H. Aono, J. Yamauchi, J. Shibayama, and H. Nakano, "Propagation loss of bent light-guiding metal line loaded on embedded dielectric core," Electron. Lett., vol. 41, no. 6, pp. 319-320, Mar. 2005.

[19] J. Shibayama, T. Yamazaki, J. Yamauchi, and H. Nakano, "Eigenmode analysis of a light-guiding metal line loaded on a dielectric substrate using the imaginary-distance beam-propagation method," J. Lightw. Technol., vol. 23, no. 3, pp. 1533-1539, Mar. 2005.

[20] P. Berini, "Plasmon-polariton waves guided by thin lossy metal films of finite width: Bound modes of symmetric structures," Phys. Rev. B, Condens. Matter, vol. 61, no. 15, pp. 10484-10 503, Apr. 2000.

[21] J. Yamauchi, M. Sekiguchi, O. Uchiyama, J. Shibayama, and H. Nakano, "Modified finite-difference formula for the analysis of semivectorial modes in step-index optical waveguides," IEEE Photon. Technol. Lett., vol. 9, no. 7, pp. 961-963, Jul. 1997.

[22] J. Yamauchi, Propagating Beam Analysis of Optical Waveguides. Hertfordshire, U.K.: Research Studies, 2003.

[23] W. P. Huang, C. L. Xu, W. Lui, and K. Yokoyama, "The perfectly matched layer (PML) boundary condition for the beam propagation method," IEEE Photon. Technol. Lett., vol. 8, no. 5, pp. 649-651, May 1996.

[24] D. Yevick, J. Yu, W. Bardyszewski, and M. Glasner, "Stability issues in vector electric field propagation," IEEE Photon. Technol. Lett., vol. 7, no. 6, pp. 658-660, Jun. 1995.

[25] P. B. Johnson and R. W. Christy, "Optical constants of the noble metals," Phys. Rev. B, Condens. Matter, vol. 6, no. 12, pp. 4370-4379, Dec. 1972.

[26] S. J. Al-Bader, "Optical transmission on metallic wires-fundamental modes," IEEE J. Quantum Electron, vol. 40, no. 3, pp. 325-329, Mar. 2004.

[27] M. A. Ordal, L. L. Long, R. J. Bell, S. E. Bell, R. R. Bell, R. W. Alexander, Jr., and C. A. Ward, "Optical properties of the metals Al, Co, $\mathrm{Cu}, \mathrm{Au}, \mathrm{Fe}, \mathrm{Pb}, \mathrm{Ni}, \mathrm{Pd}, \mathrm{Pt}, \mathrm{Ag}, \mathrm{Ti}$, and $\mathrm{W}$ in the infrared and far infrared," Appl. Opt., vol. 22, no. 7, pp. 1099-1119, Apr. 1983.

[28] D. Yevick and W. Bardyszewski, "Correspondence of variational finitedifference (relaxation) and imaginary-distance propagation methods for modal analysis," Opt. Lett., vol. 17, no. 5, pp. 329-330, Mar. 1992.

[29] C. L. Xu, W. P. Huang, and S. K. Chaudhuri, "Efficient and accurate vector mode calculations by beam propagation method," J. Lightw. Technol., vol. 11, no. 7, pp. 1209-1215, Jul. 1993.

[30] J. C. Chen and S. Jüngling, "Computation of higher-order waveguide modes by the imaginary-distance beam propagation method," Opt. Quantum Electron., vol. 29, no. 3, pp. S199-S205, 1994. 
[31] E. G. Neumann, "Reducing radiation loss of tilts in dielectric optical waveguides," Electron. Lett., vol. 17, no. 5, pp. 369-371, May 1981.

[32] T. Shiina, K. Shiraishi, and S. Kawakami, "Waveguide-bend configuration with low-loss characteristics," Opt. Lett., vol. 11, no. 11, pp. 736-738, Nov. 1986.

[33] M. Sanagi and M. Nakajima, "An optical waveguide bend with enhanced curvature and the optimization of its structure," Fiber Integr. Opt., vol. 9, no. 4, pp. 329-345, 1990.

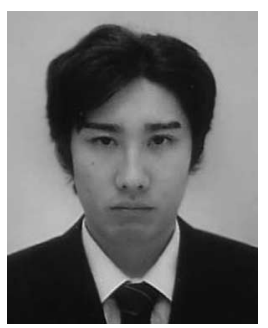

Tomohide Yamazaki (S'05) was born in Tokyo, Japan, on May 1, 1979. He received the B.E. and M.E. degrees from Hosei University, Tokyo, in 2003 and 2005 , respectively, where he is currently working toward the Dr.E. degree.

Since 2005, he has been a Research Fellow with the Japan Society for the Promotion of Science. His research interests include the numerical analysis of optical waveguides.

Mr. Yamazaki is a student member of the Institute of Electronics, Information, and Communication Engineers (IEICE) of Japan.

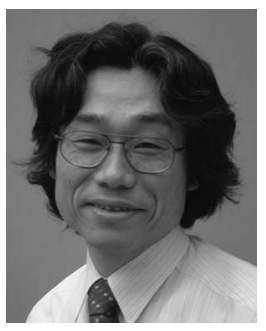

Junji Yamauchi (M'84) was born in Nagoya, Japan, on August 23, 1953. He received the B.E., M.E., and Dr.E. degrees from Hosei University, Tokyo, Japan, in 1976, 1978, and 1982, respectively.

From 1984 to 1988 , he served as a Lecturer with the Electrical Engineering Department of Tokyo Metropolitan Technical College. Since 1988, he has been a member of the faculty of Hosei University, Tokyo, where he is currently a Professor of electronic informatics. His research interests include optical waveguides and circularly polarized antennas. He is the author of Propagating Beam Analysis of Optical Waveguides (Baldock, Hertfordshire, U.K.: Research Studies, 2003).

Dr. Yamauchi is a member of the Optical Society of America and the Institute of Electronics, Information, and Communication Engineers of Japan.

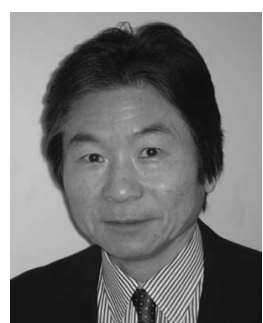

Hisamatsu Nakano (M'75-SM'87-F'92) was born in Ibaraki, Japan, on April 13, 1945. He received the B.E., M.E., and Dr.E. degrees in electrical engineering from Hosei University, Tokyo, Japan, in 1968 1970, and 1974, respectively.

Since 1973, he has been a member of the faculty of Hosei University, where he is currently a Professor of electronic informatics. His research topics include numerical methods for low- and high-frequency antennas and optical waveguides. He has published more than 200 refereed journal papers, more than 200 international symposium papers, and more than 750 national symposium papers. He is the author of the book entitled Helical and Spiral Antennas (New York: Research Studies, Wiley, 1987) and is the coauthor of the Analysis Methods of Electromagnetic Wave Problems, Volume Two (Norwood, MA: Artech House, 1986). In addition, he is also the author of the Helical and Spiral Antennas, Encyclopedia of Communications (New York: Wiley, 2002).

Dr. Nakano received the IEE International Conference on Antennas and Propagation Best Paper Award and the IEEE TRANSACTIONS ON ANTENNAS AND PRopagation Best Application Paper Award (H. A. Wheeler Award) in 1989 and 1994, respectively. In 1992, he was elected an IEEE Fellow for contributions to the design of spiral and helical antennas. In 2001, he received the Award of Distinguished Technical Communication (from the Society for Technical Communication, USA) and the Science and Technology Progress Award (from Hangzhou, China). He is an Associate Editor of several journals and magazines, such as Electromagnetics, the IEEE Antennas and Propagation Magazine, IEEE ANTENNAS AND WiRELESS PROPAGATION LETTERS, and Asian Information-Science-Life. 